tensors with $k>2 j_{\max }$.

As an example of such a process we may consider the $\mathrm{B}^{10}(d, p) \mathrm{B}^{11 *}(9.19 \mathrm{MeV})$ reaction which is known to proceed predominantly via an $l_{n}=0$ stripping process hence with $j=\frac{1}{2}$. On the other hand, a heavy-particle stripping contribution to this reaction involving pickup of $\mathrm{Be}^{9}$ by the deuterons requires $J=\frac{5}{2}$. In consequence only effects of target polarization of rank 1 are effective if pure $j=\frac{1}{2}$ direct stripping occurs, while tensors up to rank 5 can contribute if heavy-particle stripping is involved.

The magnitudes of the tensors $\epsilon_{k \kappa}\left(a ; \theta^{\prime}\right)$ and $\rho_{k \kappa}\left(b ; \theta^{\prime}\right)$ are bounded by expressions which depend on $j$. If $j$ is unique and $k=1$, and the first rank polarization is characterized by $P(a)=\langle a\rangle / \hat{a}$, then considering the components of polarization normal to the plane,

$$
\frac{d \sigma}{d \Omega}=\left(\frac{d \sigma}{d \Omega}\right)_{0}\left[1+\frac{3 a}{a+1} \cdot P(a) \cdot P^{d}(a)\right]
$$

where

$$
P^{d}(a) \leqslant[|b(b+1)-a(a+1)-j(j+1)|] / 2 a(j+1) .
$$

The upper bounds of $P^{d}(a)$ for $j=\frac{1}{2}$ and $\frac{3}{2}$ are given in the following table.

\begin{tabular}{ccc}
\hline$a+\frac{1}{2}$ & $\frac{1}{2}$ & $\frac{3}{2}$ \\
\hline$a-\frac{1}{2}$ & $\frac{1}{3}$ & $|3-a| / 5 a$ \\
$\frac{1}{3}[(a+1) / a]$ & $(a+4) / 5 a$
\end{tabular}

It may be demonstrated that, provided $j$ is unique, a series of measurements with polarized projectiles incident on polarized targets is sufficient to overdetermine the reaction amplitudes $Z_{j \zeta} \sigma_{2} \sigma_{1}\left(\theta^{\prime}\right)$.

It has also been demonstrated that polarized targets may be used to determine the parities and to identify nonzero spin states of the residual nuclei. These measurements involve the observation of the reaction product parallel or antiparallel to the incident direction, where both projectile and reaction product are of zero spin and the nucleus is of integral spin. The reaction processes need not be direct.

Moreover, detailed study with polarized targets will serve to test for small $j$-admixtures in direct reactions, to trace out the nondirect contributions to the reaction amplitudes, to exhibit spin-flip contributions to inelastic scattering processes, to differentiate between exchange processes involving heavy-particle stripping or knockout as well as to confirm nuclear clustering as involved in heavy-particle stripping.

It is clear that the nuclear information derivable from studies with polarized targets provides marked incentive for these studies despite the very considerably increased experimental complexity involved.

\footnotetext{
${ }^{*}$ This work was supported in part by the U. S. Atomic Energy Commission.

${ }^{\dagger}$ On leave from the Physics Department, The University, Manchester, England.

${ }^{1}$ See, for example, L. D. Roberts and J. W. T. Dabbs, Ann。Rev。Nuclear Sci 。11, 175 (1961).

${ }^{2}$ See, for example, L. J. B. Goldfarb and R. C. Johnson, Nuclear Phys。18, 353 (1960) and 21, 462 (1960); R. H. Bassel, R. M. Driski, and G。R.Satchler, Oak Ridge National Laboratory Report ORNL 3240, 1962 (unpublished).

${ }^{3} \mathrm{G}$. R. Satchler, Proceedings of the Rutherford Jubilee Conference, Manchester, 1961, edited by J.B. Birks (Heywood, London, 1961).

${ }^{4}$ F. Coester and J. M. Jauch, Helv. Phys. Acta 26, 3 (1953).
}

\title{
HIGH-ENERGY PROTON-PROTON DIFFRACTION SCATTERING
}

\author{
A. N. Diddens, E. Lillethun, G. Manning, ${ }^{*}$ A. E. Taylor, ${ }^{*}$ T. G. Walker, $\dagger$ and A. M. Wetherell \\ CERN, Geneva, Switzerland \\ (Received July 2, 1962)
}

This Letter reports an experiment measuring the proton-proton elastic scattering cross section at laboratory angles of about 10, 20, 30, 40, and $50 \mathrm{mrad}$ with initial momenta of $12.1,15.5$, 18.6, 21.4, and $26.2 \mathrm{GeV} / c$.

The beam, quasi-elastically scattered at 20 mrad from a Be target in the CERN proton synchrotron, was collimated to be $8 \times 1.5 \mathrm{~cm}^{2}$ at the hydrogen target, and had a divergence of
$0.6 \mathrm{mrad}$ horizontally and $2 \mathrm{mrad}$ vertically. The total intensity was $\approx 5 \times 10^{5}$ protons per pulse. Its momentum spectrum consisted of a peak at a momentum slightly below that for the circulating proton beam followed by a tail reaching about $1 \mathrm{GeV} / c$ down. The peak contained typically $70 \%$ of the protons in a momentum interval smaller than $200 \mathrm{MeV} / c$.

An 18-cm long liquid hydrogen target was used. 
The scattered particles were momentum analyzed with $4 \mathrm{~m}$ of magnetic field and detected at each angle with a threefold scintillation counter telescope. The momentum resolution was typically $5 \%$ and the solid angle of acceptance of the order of $10^{-5} \mathrm{sr}$.

The beam was continuously monitored with two small scintillation counters in coincidence. In order to obtain the total flux, these counters were scanned across the beam and the resulting numbers integrated.

Figure 1 shows two typical momentum spectra after corrections for background and accidental coincidences. Curve $A$ gives geometrical resolution function with the input momentum spectrum folded in. Curve $B$ shows the relative intensities of elastically and inelastically scattered protons used to obtain a fit to the experimental data (curve $C$ ). The relative intensities in $B$ were consistent with the results obtained from an experiment with a momentum resolution of about
$1 \% .^{1}$ The unfolding procedure was necessary only for the higher momentum transfers. The elastic differential cross sections obtained are listed in Table I.

The results are plotted in Fig. 2 in terms of $\left[\left(4 \pi / \sigma_{\left.T^{k}\right)^{2}(d \sigma / d \Omega)}\right]_{\text {c.m. }}\right.$. as a function of $|t|$ (four momentum transfer). ${ }^{2} \sigma_{T}$ is the total protonproton cross section and $k$ is the wave number of the protons in the center-of-mass system (c.m.). Only relative errors between different angles within each momentum are shown. These errors are mainly due to statistics and uncertainties in the resolution functions. The absolute errors, listed in the table, are larger because of uncertainties in the determination of the flux and the momentum spectrum of the incoming beam.

According to the optical theorem for a purely imaginary forward scattering amplitude, for $t=0,\left[\left(4 \pi / \sigma_{T^{k}}\right)^{2}(d \sigma / d \Omega)\right]_{\mathrm{c} . \mathrm{m} .}=1$.

A linear extrapolation to $t=0$ has been made

Table I. Differential elastic proton-proton scattering cross sections. $\boldsymbol{P}_{0}, \Theta$, and $-t$ are momentum of incoming proton, scattering angle, and (four-momentum transfer), ${ }^{\mathrm{a}}$ respectively.

\begin{tabular}{|c|c|c|c|c|c|c|c|c|}
\hline $\begin{array}{c}\left(F_{0}\right) \\
(\mathrm{GeV} / \mathrm{lab})\end{array}$ & $\begin{array}{c}\Theta_{\text {lab }} \\
(\mathrm{mrad})\end{array}$ & $\Theta_{\text {c.m. }}$ & $\begin{array}{c}-t \\
(\mathrm{GeV} / c)^{2}\end{array}$ & $\begin{array}{l}\frac{d \sigma}{d \Omega} \mathrm{lab} \\
(\mathrm{b} / \mathrm{sr})\end{array}$ & $\begin{array}{l}\frac{d \sigma}{d \Omega_{\mathrm{c} \cdot \mathrm{m}}} \\
(\mathrm{mb} / \mathrm{sr})\end{array}$ & {$\left[\left(\frac{4 \pi}{\sigma_{T}^{k}}\right)^{2} \frac{d \sigma}{d \Omega}\right]_{\mathrm{c} . \mathrm{m} .}$} & $\begin{array}{l}\text { Relative errors with- } \\
\text { in one momentum } \\
(\%)\end{array}$ & $\begin{array}{c}\text { Absolute } \\
\text { errors } \\
(\%)\end{array}$ \\
\hline \multirow[t]{5}{*}{12.1} & 8.8 & 2.66 & 0.011 & 4.03 & 146 & 1.10 & 11 & 19 \\
\hline & 19.1 & 5.73 & 0.052 & 2.60 & 96 & 0.70 & 8 & 16 \\
\hline & 29.1 & 8.72 & 0.121 & 1.20 & 45 & 0.33 & 5 & 16 \\
\hline & 39.1 & 11.75 & 0.219 & 0.58 & 21.3 & 0.16 & 5 & 16 \\
\hline & 49.1 & 14.7 & 0.342 & 0.20 & 5.9 & 0.057 & 6 & 16 \\
\hline \multirow[t]{5}{*}{15.5} & 8.9 & 3.02 & 0.019 & 5.79 & 165 & 0.96 & 12 & 18 \\
\hline & 18.9 & 6.40 & 0.086 & 2.63 & 76 & 0.44 & 10 & 15 \\
\hline & 28.9 & 9.71 & 0.195 & 0.92 & 27 & 0.16 & 6 & 15 \\
\hline & 38.9 & 13.25 & 0.364 & 0.22 & 6.4 & 0.038 & 8 & 16 \\
\hline & 48.9 & 16.50 & 0.563 & 0.036 & 1.1 & 0.0059 & 15 & 21 \\
\hline \multirow[t]{5}{*}{18.6} & 10.8 & 3.82 & 0.036 & 7.93 & 209 & 0.87 & 12 & 22 \\
\hline & 20.8 & 7.30 & 0.134 & 2.46 & 66 & 0.28 & 6 & 20 \\
\hline & 30.8 & 10.7 & 0.290 & 0.46 & 12.6 & 0.051 & 6 & 20 \\
\hline & 40.8 & 14.4 & 0.520 & 0.059 & 1.6 & 0.0068 & 15 & 24 \\
\hline & 50.8 & 17.8 & 0.794 & 0.009 & 0.25 & 0.0011 & 20 & 27 \\
\hline \multirow[t]{5}{*}{21.4} & 8.3 & 3.29 & 0.032 & 8.65 & 182 & 0.75 & 12 & 21 \\
\hline & 18.6 & 7.30 & 0.155 & 1.95 & 43 & 0.175 & 8 & 18 \\
\hline & 28.6 & 11.18 & 0.364 & 0.29 & 6.4 & 0.025 & 7 & 19 \\
\hline & 38.6 & 15.30 & 0.680 & 0.024 & 0.52 & $0.0 \times 22$ & 10 & 20 \\
\hline & 48.6 & 19.0 & 1.055 & 0.0020 & 0.045 & 0.00018 & 30 & 34 \\
\hline \multirow[t]{4}{*}{26.2} & 9.5 & 4.21 & 0.064 & 11.3 & 189 & 0.65 & 13 & 22 \\
\hline & 19.5 & 8.60 & 0.268 & 1.31 & 22.4 & 0.078 & 7 & 21 \\
\hline & 29.5 & 12.88 & 0.596 & 0.072 & 1.24 & 0.0043 & 10 & 22 \\
\hline & 39.5 & 17.03 & 1.042 & 0.0041 & 0.075 & 0.00025 & 20 & 28 \\
\hline
\end{tabular}

\footnotetext{
${ }^{\mathrm{a}}$ See reference 2 .
} 


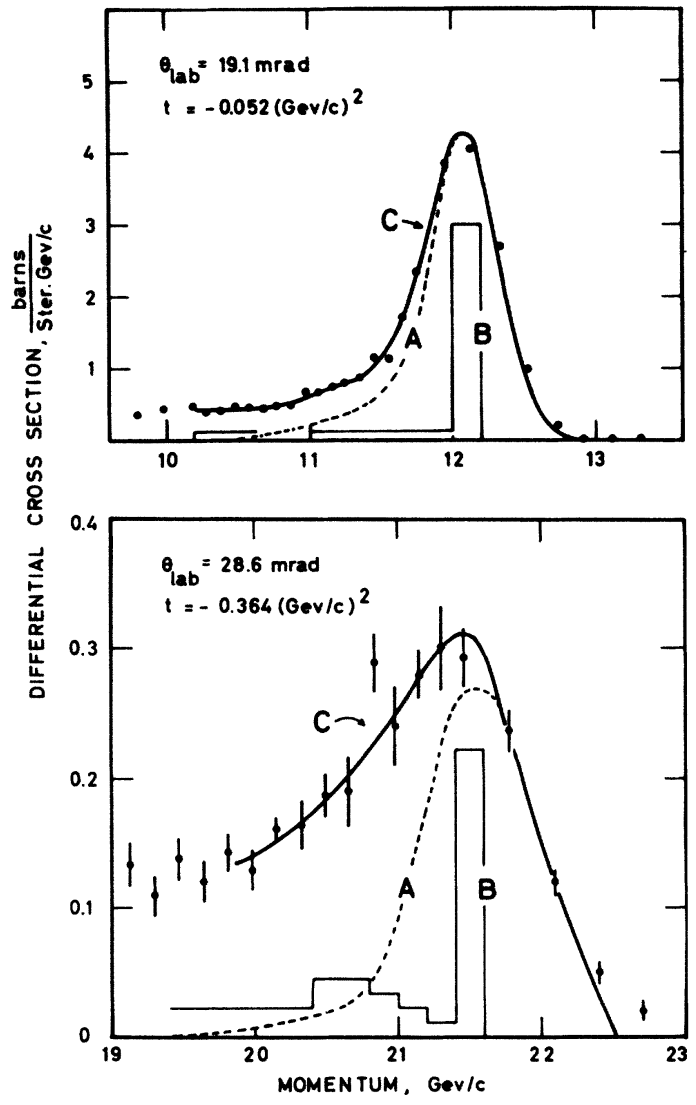

FIG. 1. Typical momentum spectra from protonproton scattering at initial momenta of 12.1 and 21.4 $\mathrm{GeV} / c$ at laboratory scattering angles of 19.1 and 28.6 mrad. Curves $A, B$, and $C$ are explained in the text.

using the data at the two smallest $|t|$ values at each momentum. An average value of $1.2 \pm 0.2$ was obtained. The initial slopes of the curves in Fig. 2 are all consistent with a $t$-dependence of $\exp (-11|t|)$.

The data are used in conjunction with other similar data in a Regge pole analysis presented in the following Letter.

The total elastic scattering cross sections shown in Table II have been obtained by integrating under the curves in Fig. 2. The results are plotted in Fig. 3 together with those of other measurements at momenta higher than $2 \mathrm{GeV} / c .^{2-8}$ We should like to thank L. Bird, R. Donnet,

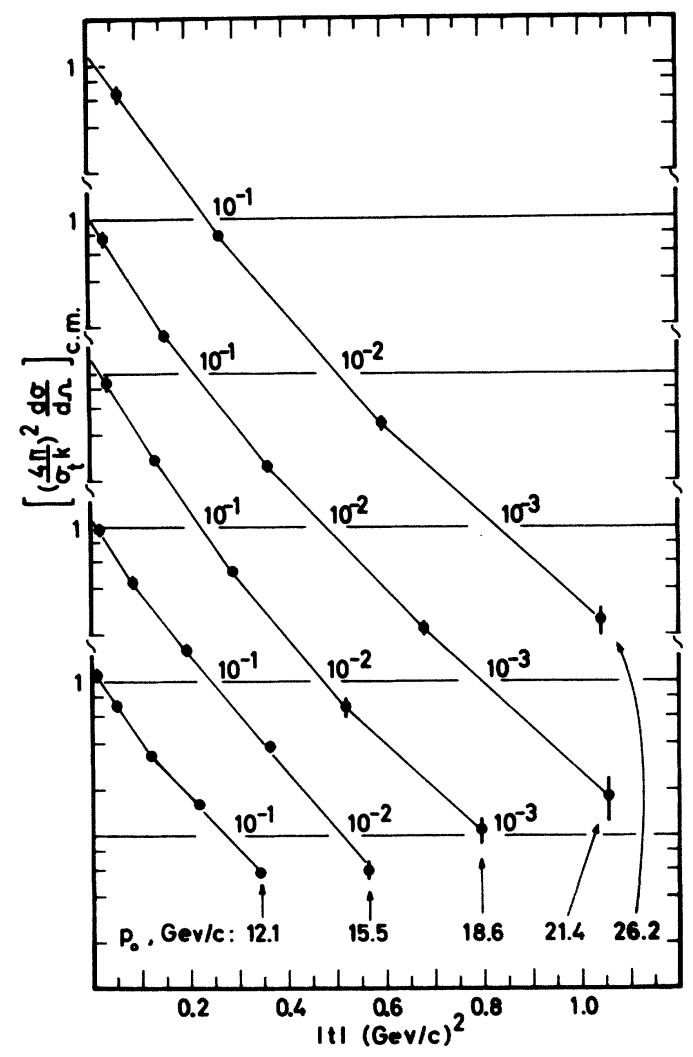

FIG. 2. Normalized differential elastic cross sections. Each distribution is displaced by one decade. Only relative errors are shown.

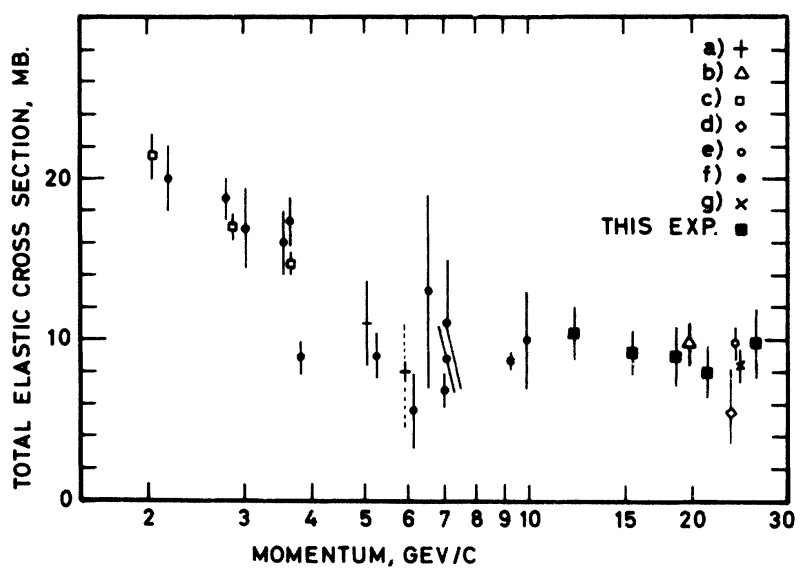

FIG. 3. Total elastic proton-proton cross sections. For a-g, see references $2-8$, respectively.

Table II. Total elastic proton-proton scattering cross section.

\begin{tabular}{|c|c|c|c|c|c|}
\hline$P_{0}(\mathrm{GeV} / c)$ & 12.1 & 15.5 & 18.6 & 21.4 & 26.2 \\
\hline Elastic $\sigma_{T}(\mathrm{mb})$ & $10.4 \pm 1.7$ & $9.2 \pm 1.4$ & $9.0 \pm 1.8$ & $8.0 \pm 1.6$ & $9.8 \pm 2.2$ \\
\hline
\end{tabular}


and C. A. Stahlbrandt for their assistance throughout the experiment, and the Proton Synchrotron Machine Division for their cooperation.

\footnotetext{
${ }^{*}$ On leave of absence from the Atomic Energy Research Establishment, Harwell, England.

†On leave of absence from the National Institute for Research in Nuclear Science, Harwell, England.

${ }^{1}$ G. Cocconi, A. N. Diddens, E. Lillethun, G. Manning, A. E. Taylor, T. G. Walker, and A. M. Wetherell, Phys. Rev. Letters 7, 450 (1961).

${ }^{2}$ M. H. Blue, J. J. Lord, J. G. Parks, and C. H. Tsao, Phys. Rev. 125, 1386 (1962); C. H. Tsao, J. G. Parks, and J. J. Lord, Bull. Am. Phys. Soc. 6, 343 (1961).

${ }^{3} \mathrm{~V}$. Bull and D. Garbutt (private communication).

${ }^{4}$ G. B. Chadwick, G. B. Collins, P. J. Duke, T. Fu-
}

jii, N. C. Hien, and F. Turkot (private communication, to be published).

${ }^{5}$ G. Cvijanovich, B. Dayton, P. Egli, B. Klaiber, W. Koch, M. Nikolić, R. Schneeberger, H. Winzeler, J. C. Combe, W. M. Gibson, W. O. Lock, M. Schneeberger, and G. Vanderhaeghe, Nuovo cimento 20, 1012 (1961).

${ }^{6}$ P. Dodd, M. Jobes, J. Kinson, B. Tallini, B. R. French, H. J. Sherman, I. O. Skillicorn, W. T. Davies, M. Derrick, and D. Radojicic, in Proceedings of the International Conference on Elementary Particles, 1961 (Commissariat a l' Energie Atomique, Saclay, Gif-surYvette, 1962), p. 433.

${ }^{7}$ E. Marquit, Institute of Nuclear Research, Polish Academy of Sciences, Warsaw, Internal Report 255/VI, 1961 (unpublished). See this review paper for other references.

${ }^{8}$ D. R. O. Morrison (private communication).

\title{
HIGH -ENERGY PROTON-PROTON SCATTERING
}

\author{
A. N. Diddens, E. Lillethun, G. Manning, ${ }^{*}$ A. E. Taylor ${ }^{*}$ T. G. Walker, ${ }^{\dagger}$ and A. M. Wetherell \\ CERN, Geneva, Switzlerand \\ (Received July 2, 1962)
}

This Letter reports measurements of $p-p$ differ ential elastic cross sections for large momentum transfers. These data together with results of other measurements are analyzed by means of a single Regge pole term.

The new data presented in this Letter were obtained in a manner similar to that described by Cocconi et al. ${ }^{1}$ except that the scattering angle was changed to $110 \mathrm{mrad}$. This extended the results to a value of $-5.5(\mathrm{GeV} / c)^{2}$ for the square of the four-momentum transfer $t$.

The cross section for $\mathrm{C}^{12}(p, 3 p 3 n) \mathrm{Be}^{7}$ is used in the $\mathrm{CH}_{2}-\mathrm{C}$ subtraction procedure to obtain the $p-p$ cross sections. A recent measurement ${ }^{2}$ of this cross section gives a value of $7.7 \pm 0.4 \mathrm{mb}$, whereas $11 \mathrm{mb}$ had been used for the previous experiments at 56 and $60 \mathrm{mrad}^{1}$ The earlier data have been corrected accordingly, and small changes have been made to the normalization of the $\mathrm{CH}_{2}$ and $C$ spectra for the 12.99-, 15.89-, and 27.83$\mathrm{GeV} / c$ data at $\approx 56 \mathrm{mrad}$. It is these corrected values which appear in Table I, together with the cross sections from the measurements at 110 mrad.

The combined results of these measurements, together with those in the preceding Letter, ${ }^{3}$ are plotted in Fig. 1 as $\left[\left(4 \pi / \sigma_{T} k\right)^{2}(d \sigma / d \Omega)\right]_{\text {c.m. }}$. against $|t|$. This normalized cross section is equal to $(d \sigma / d t) /(d \sigma / d t)_{t=0}$ using the optical theorem for the forward scattering amplitude. Here $k$ is the appropriate c.m. wave number and the $p-p$ total cross section, $\sigma_{T}$, is taken from published values. The smallest cross section, obtained at $110 \mathrm{mrad}$ and $21.46 \mathrm{GeV} / c$, corresponds to $3 \times 10^{-32} \mathrm{~cm}^{2} / \mathrm{sr}$ at $42^{\circ}$ in the c.m. system. In this region the cross section still appears to be decreasing but with a slope $\approx \frac{1}{5}$ of that for the small angle scattering.

Figure 2 shows the normalized cross sections plotted against $s / 2 M^{2}$ for various values of $|t|$. $s$ is the square of the c.m. energy and $M$ is the nucleon mass. The cross sections for $|t|<1$ were obtained by interpolation between measured values whereas for larger $|t|$ values, direct cross sections are plotted. Data from lower energies are also included. ${ }^{4,5}$ Least-square straight-line fits have been made to the points for constant $|t|$ and the slopes of these lines exhibit a shrinking of the diffraction pattern.

Recent considerations of a Regge pole theory, ${ }^{6,9}$ and of a specific field-theoretical model, ${ }^{10}$ have suggested that the diffraction cross section can be put in the form

$$
\left(\frac{d \sigma}{d t}\right) /\left(\frac{d \sigma}{d t}\right)_{t=0}=F(t)\left(s / 2 M^{2}\right)^{2[\alpha(t)-1]} \text {. }
$$

The functional dependence of both $F(t)$ and $\alpha(t)$ 